\title{
A Novel Homozygous SLC2A9 Mutation Associated with Renal-Induced Hypouricemia
}

\author{
Martin Windpessl ${ }^{\mathrm{a}} \quad$ Marco Ritelli $^{\mathrm{b}} \quad$ Manfred Wallner ${ }^{\mathrm{a}} \quad$ Marina Colombi $^{\mathrm{b}}$ \\ ${ }^{a}$ Division of Nephrology, Fourth Department of Internal Medicine, Klinikum Wels-Grieskirchen, Wels, Austria; \\ ${ }^{b}$ Division of Biology and Genetics, Department of Molecular and Translational Medicine, University of Brescia, \\ Brescia, Italy
}

\section{Key Words}

Hypouricemia - Glucose transporter 9.SLC2A9 - Acute kidney injury

\begin{abstract}
Background: Hereditary renal hypouricemia (RHUC) is a genetically heterogenous disorder characterized by defective uric acid (UA) reabsorption resulting in hypouricemia and increased fractional excretion of UA; acute kidney injury $(\mathrm{AKI})$ and nephrolithiasis are recognized complications. Type 1 (RHUC1) is caused by mutations in the SLC22A12 gene, whereas RHUC2 is caused by mutations in the SLC2A9 gene. Patient ethnicity is diverse but only few Caucasian families with an SLC2A9 mutation have been reported. Methods: The current report describes the clinical history, biochemical and molecular genetics findings of a native Austrian family with RHUC2. The propositus presented with 2 episodes of exercise-induced AKI and exhibited profound hypouricemia. Mutational screening of the SLC22A12 and SLC2A9 genes was performed. Results: The molecular analyses revealed the homozygous C.512G >A transition that leads to the p.Arg171His missense substitution in SLC2A9, confirming the diagnosis of RHUC2. Segregation study of the causal mutation revealed that the mother and elder sister were heterozygous carriers, whereas the younger sister was found to be homozygous. Conclusion: We report the identification of a novel mutation
\end{abstract}

\section{KARGER}

E-Mail karger@karger.com

www.karger.com/ajn in SLC2A9 as the cause of RHUC2 in a native Austrian family. We show that glucose transporter 9 mutations cause severe hypouricemia in homozygous individuals and confirm the high risk of $\mathrm{AKI}$ in male individuals harbouring these mutations. In our literature review, we provide an overview of the putative underlying pathophysiology, potential renal complications, findings on kidney biopsy as well as potential long-time renal sequelae.

(c) 2016 S. Karger AG, Basel

\section{Introduction}

Hereditary renal hypouricemia (RHUC) is a genetically heterogenous disorder caused by recessive mutations in genes encoding distinct urate transporters in the proximal renal tubule. The resultant defective urate reabsorption causes increased urate clearance with very low serum uric acid (UA) levels $(<2 \mathrm{mg} / \mathrm{dl})$ [1]. While affected individuals frequently remain asymptomatic, a predisposition for nephrolithiasis and acute kidney injury (AKI), often following exercise, has been recognized.

Mutations in 2 genes account for virtually all reported cases, with notable exceptions $[2,3]$. In a majority of patients, the defect is caused by loss-of function mutations in the SLC22A12 gene that codes for the urate transporter URAT1 (RHUC1). In addition, familial hypouricemia 

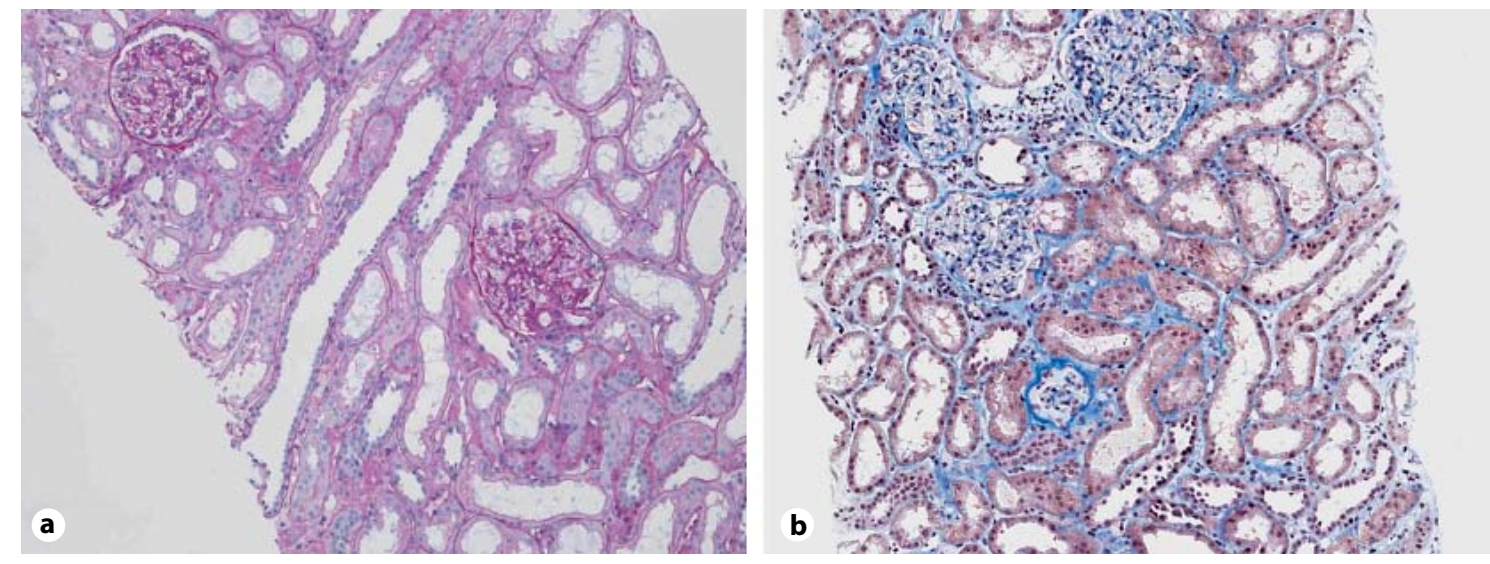

Fig. 1. Renal biopsy showing mild signs of acute tubular injury with tubular ectasia with flattening of epithelia cells (focal interstitial oedema) with very few scattered interstitial inflammatory cells, no casts or precipitations within tubuli (and no glomerular lesions): a periodic acid-Schiff; b trichrome acidic fuchsin orange-G.

has also been described in patients harbouring homozygous or compound heterozygous mutations in the SLC2A9 gene, which encodes another key player in UA homeostasis, glucose transporter 9 (GLUT9; RHUC2). Patient ethnicity is diverse and includes Japanese, Chinese, Arab, Ashkenazi-Jewish, Anglo-Saxon, Greek, and Czech; to date, only few Caucasian families with a GLUT9 mutation have been reported [4-7].

We describe a young native Austrian man who experienced 2 episodes of exercise-induced AKI, exhibited profound hypouricemia with markedly increased fractional excretion (FE) of UA and was found to be homozygous for a novel missense mutation in SLC2A9. While the mother and elder sister tested heterozygous for the mutation, the younger sister carried the mutation in homozygosity and displayed extremely low serum UA levels; however, she has not experienced any episodes of AKI.

\section{Case Report}

A previously well 16-year-old Caucasian male was admitted because of sudden onset of bilateral loin pain, nausea and vomiting. There was no history of nephrolithiasis and family history was unremarkable. He was on no regular medications and did not smoke or use illicit drugs. The previous day, he had engaged in $20 \mathrm{~min}$ of barbell-training. On admission, the patient was euvolemic, afebrile; blood pressure was $144 / 82 \mathrm{~mm} \mathrm{Hg}$ and pulse rate 96 beats per minute. Apart from mild abdominal tenderness, physical examination was unremarkable.

Pertinent laboratory results were as follows: serum creatinine $2.1 \mathrm{mg} / \mathrm{dl}$, creatine kinase (CK) 1,503 U/l, lactate dehydrogenase $250 \mathrm{U} / \mathrm{l}$; full blood count was normal. Urinalysis showed trace of protein (+) but no cells, casts, haemoglobin or myoglobin; urinary $\mathrm{pH}$ was 6. Serologic data (anti-nuclear antibody, hepatitis B and C, human immunodeficiency virus and complement) were all normal. Toxicology screen was negative. A renal ultrasound showed normal-sized kidneys with no evidence of obstruction. Within 3 days, serum creatinine peaked at $4.4 \mathrm{mg} / \mathrm{dl}$, while CK normalized. UA, which was not done initially, was $1.1 \mathrm{mg} / \mathrm{dl}$ at that time. A kidney biopsy was performed and showed only subtle signs of acute tubular injury without intratubular UA precipitation (fig. 1a, b). Renal function recovered spontaneously and profound hypouricemia $(0.2 \mathrm{mg} / \mathrm{dl}$ at discharge) became evident. Blood results from a previous admission 3 years earlier showed normal serum creatinine $(0.8 \mathrm{mg} / \mathrm{dl})$ but UA of $0.1 \mathrm{mg} / \mathrm{dl}$. No secondary causes of low UA were evident. FE-UA was very high $(126 \%$, normal range $7-12 \%)$ in the acute phase.

In view of the marked hypouricemia and high FE-UA, a diagnosis of RHUC was suspected, which led us to perform mutational screening of the SLC22A12 and SLC2A9 genes, as previously described [8]. The molecular analyses revealed the homozygous c.512G $>$ A transition (seq. ref.: NM_020041.2) that leads to the p. Arg171Hismissensesubstitution(GLUT9L, seq.ref.:NP_064425.2) in SLC2A9, thus confirming the diagnosis of the RHUC type 2 (fig. 2). Interestingly, the Arg171 residue has been previously reported by Dinour et al. [9] to be substituted by a Cys residue in 3 siblings, born of consanguineous Israeli-Arab parents, with RHUC2 and without complications. In vitro functional expression studies showed that the p.Arg171Cys mutant protein had significantly reduced UA transport activity ( $16 \%$ of control values). Furthermore, homology modelling indicated that the mutation occurred in the inner channel that expels UA from the cytoplasmic to extracellular regions [9].

The serum UA levels were screened in other family members and were $2.7 \mathrm{mg} / \mathrm{dl}$ (mother), $2.7 \mathrm{mg} / \mathrm{dl}$ (elder sister) and $0.1 \mathrm{mg} / \mathrm{dl}$ (younger sister); FE-UA was 22, 14.2 and $>150 \%$, respectively. The father could not be contacted. Segregation study of the causal mutation, performed for all available relatives, revealed that the mother and elder sister were heterozygous carriers (fig. 2), whereas the younger sister was found to be homozy- 


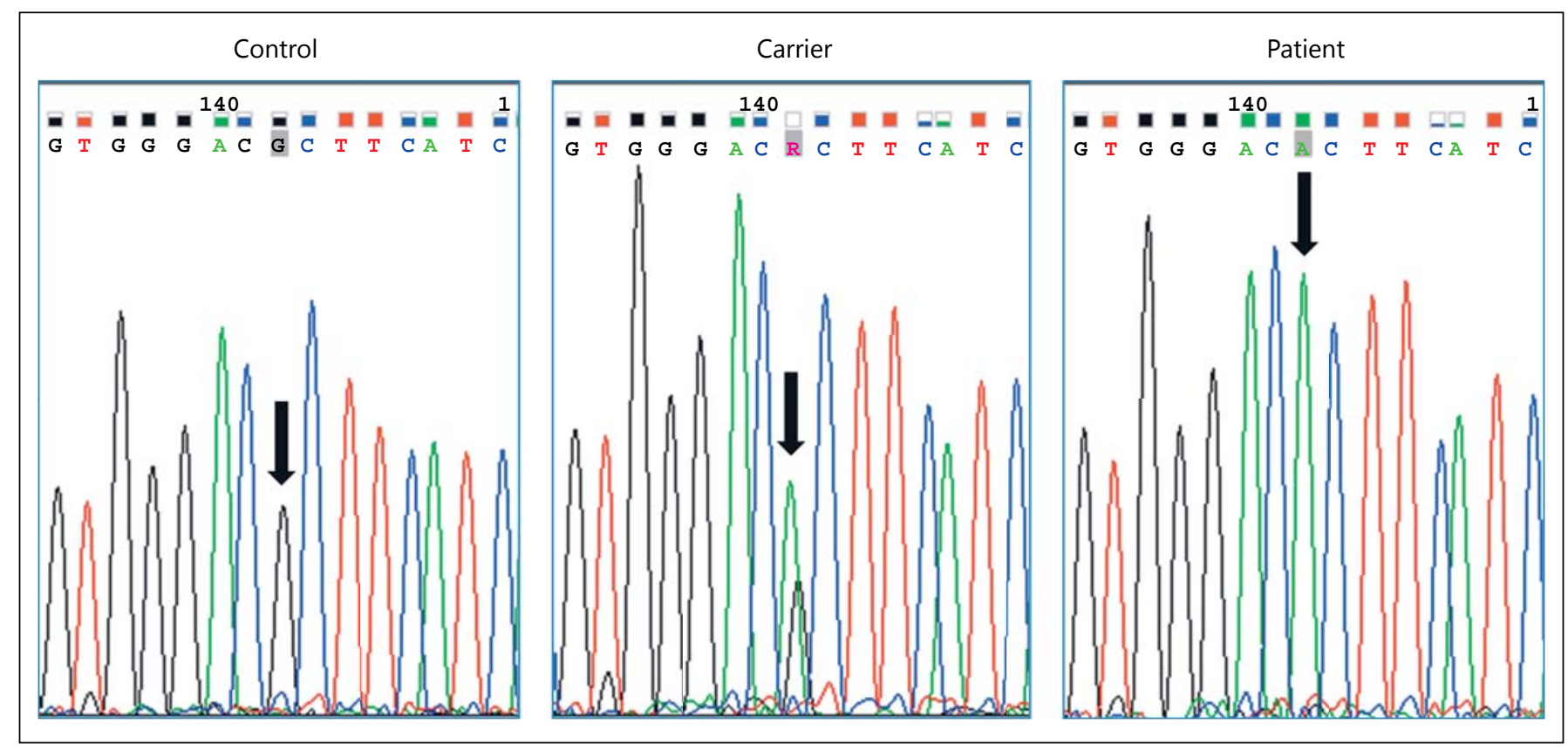

Fig. 2. Molecular characterization. Sequence chromatogram showing the position of the novel c.512G > A transition (NM_020041.2, GLUT9L) that corresponds to c.425G>A (NM_00100290.1, GLUT9S), leading to the p.Arg171His missense mutation (NP_064425.2, GLUT9L) and to p.Arg142His (NP_001001290.1,
GLUT9S). The mutation was found in homozygosity in the index patient and his younger sister and in heterozygosity in his mother and elder sister; the father was not available for genetic testing. Mutations are annotated according to HGVS nomenclature (http://www.hgvs.org/mutnomen). gous, in agreement with her very low UA levels. However, despite an active lifestyle, at the age of 24 , she remained asymptomatic until this date.

In contrast, and 9 months after his initial presentation, the index patient was readmitted with abdominal pain and myalgia of the upper arms. The previous day, he had moved furniture for several hours. At presentation, only 1 day after the onset of symptoms, his serum creatinine was $3.9 \mathrm{mg} / \mathrm{dl}$. Again, no structural abnormalities were detected on renal ultrasound. Urinary dipstick showed protein (quantified at $0.8 \mathrm{~g}$ at that time) but no haemoglobinuria; urinary $\mathrm{pH}$ was 6 . No UA crystals were found under urine microscopy. Kidney function and proteinuria normalized spontaneously. On follow-up, UA remained between 0.1 and $0.2 \mathrm{mg} / \mathrm{dl}$ and FE-UA was calculated repeatedly $>150 \%$. By avoidance of strenuous exercise no further episode of exercise-induced AKI has occurred to date.

The presence of a novel mutation in homozygosity is suggestive of consanguinity, though the family pedigree could not be defined with certainty.

\section{Discussion}

In humans, urate is the end product in purine metabolism and it exerts important oxidative defence. Its serum concentration is governed by hepatic production and predominantly renal removal. Urate is filtered freely by the glomerulus and is almost completely reabsorbed in the proximal tubule; consequently, the kidney plays a dominant role in maintaining serum urate levels [1].

Hypouricemia is arbitrarily defined as a serum urate concentration of $<2 \mathrm{mg} / \mathrm{dl}$. While it can result from decreased UA production, it is usually due to renal urate wasting [4]. Following Erley's description in 1989 of a young Turkish man who experienced exercise-induced AKI requiring transient dialysis and was later found to be profoundly hypouricemic, it became evident that RHUC constitutes not just a mere biochemical curiosity [10]. The ensuing years saw an increasing number of reports of RHUC patients presenting with acute kidney failure [11]. However, the underlying molecular defect remained obscure until Enomoto et al. [12] reported the identification of the first kidney specific urate transporter URAT1 (SLC22A12) in 2002. Inactivating mutations in this gene have been subsequently found in individuals with idiopathic RHUC and $>100$ patients have been described, the majority being Japanese [13]. However, the existence of RHUC patients without URAT1 mutations implied the presence of additional major urate regulators [1].

In 2007, GLUT9 (encoded by SLC2A9) was identified as another high capacity urate transporter [14]. GLUT 
proteins are encoded by the SLC2 genes and mediate the transport of monosaccharides and other small carbon compounds across cell membranes. To date, 14 GLUT proteins have been identified, but GLUT9 represents the only member of this family whose substrate is urate. Its 2 variants - long (GLUT9L) and short (GLUT9S) - act in tandem with URAT1 in renal reabsorption of UA [15]. While urate uptake from the tubular lumen into the cell is mediated by URAT1 and by apical GLUT9S, GLUT9L is the only major urate efflux transporter at the basolateral membrane. Hence, loss of function of GLUT9 precludes UA reabsorption by all of the apical transporters through complete inhibition of UA efflux from the cell [9].

Shortly thereafter, mutations in SLC2A9 were described in hypouricemic patients, rendering SLC2A9 another causative gene linked to RHUC [16]. When compared to patients with URAT1 mutations, individuals harbouring homozygous GLUT9 mutations exhibit more pronounced hypouricemia with FE-UA often $>150 \%$, and appear to be more vulnerable for exercise-induced AKI and nephrolithiasis $[13,16]$. Subjects with heterozygous GLUT9 mutations show a wider spectrum of serum UA levels, ranging from 2.0 to $4.5 \mathrm{mg} / \mathrm{dl}$, whereas FE-UA values range from 3.2 to $21.7 \%$ in one study from Israel [16]; these authors concluded that haploinsufficiency results in mild hypouricemia. The recent report of a 54-yearold Czech woman with a novel heterozygous missense mutation in the SLC2A9 gene, exhibiting low serum UA levels $(1.16-1.78 \mathrm{mg} / \mathrm{dl})$ and FE-UA of $17.7 \%$, is in line with this observation; functional study of the identified mutation showed a significant decrease in urate uptake [7]. We have previously summarized the molecular and clinical features of all RHUC2 patients reported until 2014 [8], and only a few new cases were published in the meanwhile $[6,7,17,18]$.

Several mechanisms have been proposed to explain the pathophysiology of AKI in RHUC. Although Erley's description demonstrated urate nephropathy as the histological correlate of acute renal failure, further reports did not substantiate this finding [19]; indeed, a majority of renal biopsies has shown acute tubular injury without intratubular UA precipitation [20]. However, luminal microcrystals will not be inevitably evident on histology. As discussed in our previous report, the UA precipitation hypothesis proposes that prolonged or severe exercise can be associated with an elevation in UA production and urinary excretion [8]. If this condition is associated with hypovolemia, increased urine concentration and low urine $\mathrm{pH}$, it favours the precipitation and crystallization of UA. Notably, a recent paper on 'heat stress nephropathy' dis- cusses the potential detrimental effects of cyclic, exerciseinduced uricosuria in the pathogenesis of Mesoamerican nephropathy. The authors show that high concentrations of noncrystalline UA per se, potentiated by volume depletion and urinary acidification in the course of exercise, cause renal tubular injury [21]. This concept could also apply to RHUC patients.

While the precise mechanism for kidney injury remains elusive, the role of UA as an antioxidant has increasingly garnered attention. Murakami et al. [22] were the first to suggest that oxygen-free radicals produced during exercise lead to renal tissue damage. Total serum UA and the amount mobilized into the tubular cells are very small in RHUC patients, resulting in insufficient antioxidant action in the renal cortex. As oxidative stress increases during exercise, renovascular spasm may ensue and reperfusion after exercise mimics ischemia-reperfusion injury [23]. The histological finding of interlobular artery intimal thickening in a normotensive RHUC2 patient, attributed to repeated vasoconstriction, renders further support to this pathophysiological concept, which is also backed by CT findings showing patchy wedgeshaped enhancement in renal parenchyma $[18,24]$. Interestingly, evidence for a more systemic oxidant imbalance at presentation with AKI accumulates: 3 cases of posterior reversible encephalopathy syndrome have been recently reported in RHUC1 and RHUC2 patients, and one Japanese RHUC1 individual exhibited reversible T-wave inversion on ECG; together, these reports are suggestive of cerebral and coronary artery constriction, respectively [19, 24-26]. That being said, other disorders associated with hypouricemia, such as hereditary xanthinuria, are not usually associated with AKI. Moreover, RHUC patients can remain asymptomatic throughout their life, as exemplified by an 84 -year-old subject with a homozygous SLC2A9 mutation [9]. We have previously speculated that additional genetic risk factors, for instance, sequence variations in other urate transporters, and various environmental triggers, may contribute to the development of complications [8]. Lastly, expression of URAT1 and GLUT9 is not confined to the kidney: while URAT1 is expressed by vascular smooth muscle cells, GLUT9 is also found in the brain $[25,27]$. We hypothesize that this systemic distribution might account for the non-renal complications of RHUC.

The presentation follows a remarkably stereotypical pattern. After various forms of exercise (occasionally trivial activities such as house cleaning) [20], loin pain, nausea and vomiting develop. The short time lag between exercise and symptom onset in the presentation is notewor- 
thy and in keeping with reversible vasoconstriction rather than urate nephropathy. While moderate CK elevation is commonly found, frank rhabdomyolysis is unusual.

The marked male preponderance for exercise-related AKI, with a male:female ratio of $8: 1$ in one study, has been noted before and is found consistently in the literature [8, $11,21]$. Gender-related differences in oxidative stress, attributed to estradiol, might account for this phenomenon [23].

Previous laboratory examinations, when available, may assist in RHUC diagnosis. While complex urine examination was commonly performed in patients with hypouricemia before the characterization of the major urate transporters, suggestive biochemistry and family history should prompt early molecular analysis of the 2 known causative genes, as illustrated by our reports.

Patients should be counselled to avoid strenuous exercise and ensure adequate fluid intake after exercise. Rather counterintuitively, allopurinol, a potent inhibitor of xanthine oxidase, may have a role in preventing recurrent episodes [3]. While this basically fits with the 'cyclic uricosuria'-hypothesis, (high-dose) allopurinol has also been shown to improve endothelial function by reducing vascular oxidative stress, perhaps unrelated to its effect on UA [28]. In the metabolism of xanthine to UA, free radicals are generated; it is conceivable, therefore, that allopurinol exerts its effect as a powerful antioxidant.

Although some patients require interim dialysis, the short-term prognosis of RHUC appears to be favourable.
However, recurrence of AKI has been described and there is evidence that repeated kidney injury can cause chronic structural alterations $[18,20]$; end-stage renal disease in RHUC patients has been reported [29]. Furthermore, a recent large cross-sectional population-based study from Japan demonstrated that men with hypouricemia face a higher risk of reduced kidney function [30].

As stipulated by Stiburkova et al. [13], lack of awareness of RHUC likely allows cases to go undetected. Indeed, patients have been subjected to repeat renal biopsies when hypouricemia was not noticed at first presentation [17].

To summarize, we report the identification of a novel mutation in SLC2A9 as the cause of RHUC2 in a native Austrian family. In analogy with previous reports, we show that GLUT9 mutations cause severe hypouricemia in homozygous individuals and confirm the high risk of $\mathrm{AKI}$ in male individuals harbouring these mutations.

\section{Acknowledgement}

The authors greatly appreciate Prof. Heinz Regele from the Department of Pathology, Medical University of Vienna, for the histopathology images.

\section{Disclosure Statement}

The authors declare that there is no conflict of interests.

\section{References}

1 Sperling O: Hereditary renal hypouricemia. Mol Genet Metab 2006;89:14-18.

2 Kaito H, Ishimori S, Nozu K, Shima Y, Nakanishi K, Yoshikawa N, Iijima K: Molecular background of urate transporter genes in patients with exercise-induced acute kidney injury. Am J Nephrol 2013;38:316-320.

3 Bhasin B, Stiburkova B, De Castro-Pretelt M, Beck N, Bodurtha JN, Atta MG: Hereditary renal hypouricemia: a new role for allopurinol? Am J Med 2014;127:e3-e4.

4 Stiburkova B, Ichida K, Sebesta I: Novel homozygous insertion in SLC2A9 gene caused renal hypouricemia. Mol Genet Metab 2011; 102:430-435.

5 Stiburkova B, Taylor J, Marinaki AM, Sebesta I: Acute kidney injury in two children caused by renal hypouricaemia type 2 . Pediatr Nephrol 2012;27:1411-1415.

6 Androvitsanea A, Stylianou K, Maragkaki E, Tzanakakis M, Stratakis S, Petrakis I, Giatzakis C, Daphnis E: Vanishing urate, acute kidney in- jury episodes and a homozygous SLC2A9 mutation. Int Urol Nephrol 2015;47:1035-1036.

7 Mancikova A, Krylov V, Hurba O, Sebesta I, Nakamura M, Ichida K, Stiburkova B: Functional analysis of novel allelic variants in URAT1 and GLUT9 causing renal hypouricemia type 1 and 2. Clin Exp Nephrol 2015, Epub ahead of print.

8 Jeannin G, Chiarelli N, Gaggiotti M, Ritelli M, Maiorca P, Quinzani S, Verzeletti F, Possenti S, Colombi M, Cancarini G: Recurrent exercise-induced acute renal failure in a young Pakistani man with severe renal hypouricemia and SLC2A9 compound heterozygosity. BMC Med Genet 2014; 15:3.

9 Dinour D, Gray NK, Ganon L, Knox AJ, Shalev H, Sela BA, Campbell S, Sawyer L, Shu X, Valsamidou E, Landau D, Wright AF, Holtzman EJ: Two novel homozygous SLC2A9 mutations cause renal hypouricemia type 2. Nephrol Dial Transplant 2012;27: 1035-1041.
10 Erley CM, Hirschberg RR, Hoefer W, Schaefer K: Acute renal failure due to uric acid nephropathy in a patient with renal hypouricemia. Klin Wochenschr 1989;67:308-312.

11 Yeun JY, Hasbargen JA: Renal hypouricemia: prevention of exercise-induced acute renal failure and a review of the literature. Am J Kidney Dis 1995;25:937-946.

12 Enomoto A, Kimura H, Chairoungdua A, Shigeta Y, Jutabha P, Cha SH, Hosoyamada M, Takeda M, Sekine T, Igarashi T, Matsuo $H$, Kikuchi Y, Oda T, Ichida K, Hosoya T, Shimokata K, Niwa T, Kanai Y, Endou H: Molecular identification of a renal urate anion exchanger that regulates blood urate levels. Nature 2002;417:447-452.

13 Stiburkova B, Sebesta I, Ichida K, Nakamura M, Hulkova H, Krylov V, Kryspinova L, Jahnova $\mathrm{H}$ : Novelallelic variants and evidence for a prevalent mutation in URAT1 causing renal hypouricemia: biochemical, genetics and functional analysis. Eur J Hum Genet 2013;21:1067-1073. 
14 Li S, Sanna S, Maschio A, Busonero F, Usala G, Mulas A, Lai S, Dei M, Orrù M, Albai G, Bandinelli S, Schlessinger D, Lakatta E, Scuteri A, Najjar SS, Guralnik J, Naitza S, Crisponi L, Cao A, Abecasis G, Ferrucci L, Uda M, Chen WM, Nagaraja R: The GLUT9 gene is associated with serum uric acid levels in Sardinia and Chianti cohorts. PLoS Genet 2007; 3:e194.

15 Kimura T, Takahashi M, Yan K, Sakurai H: Expression of SLC2A9 isoforms in the kidney and their localization in polarized epithelial cells. PLoS One 2014;9:e84996.

16 Dinour D, Gray NK, Campbell S, Shu X, Sawyer L, Richardson W, Rechavi G, Amariglio N, Ganon L, Sela BA, Bahat H, Goldman M, Weissgarten J, Millar MR, Wright AF, Holtzman EJ: Homozygous SLC2A9 mutations cause severe renal hypouricemia. J Am Soc Nephrol 2010;21:64-72.

17 Shen H, Feng C, Jin X, Mao J, Fu H, Gu W, Liu A, Shu Q, Du L: Recurrent exercise-induced acute kidney injury by idiopathic renal hypouricemia with a novel mutation in the SLC2A9 gene and literature review. BMC Pediatr 2014;14:73.

18 Mou LJ, Jiang LP, Hu Y: A novel homozygous GLUT9 mutation cause recurrent exerciseinduced acute renal failure and posterior reversible encephalopathy syndrome. J Nephrol 2015;28:387-392.

19 Tanaka M, Itoh K, Matsushita K, Matsushita K, Wakita N, Adachi M, Nonoguchi H, Kita- mura K, Hosoyamada M, Endou H, Tomita K: Two male siblings with hereditary renal hypouricemia and exercise-induced ARF. Am J Kidney Dis 2003;42:1287-1292.

20 Ohta T, Sakano T, Igarashi T, Itami N, Ogawa $\mathrm{T}$ : Exercise-induced acute renal failure associated with renal hypouricaemia: results of a questionnaire-based survey in Japan. Nephrol Dial Transplant 2004;19:1447-1453.

21 Roncal-Jimenez C, García-Trabanino R, Barregard L, Lanaspa MA, Wesseling C, Harra T, Aragón A, Grases F, Jarquin ER, González MA, Weiss I, Glaser J, Sánchez-Lozada LG, Johnson RJ: Heat stress nephropathy from exercise-induced uric acid crystalluria: a perspective on Mesoamerican nephropathy. Am J Kidney Dis 2016;67:20-30.

22 Murakami T, Kawakami H, Fukuda M, Furukawa S: Patients with renal hypouricemia are prone to develop acute renal failure - why? Clin Nephrol 1995;43:207-208.

23 Kaneko K, Taniguchi N, Tanabe Y, Nakano T, Hasui M, Nozu K: Oxidative imbalance in idiopathic renal hypouricemia. Pediatr Nephrol 2009;24:869-871.

24 Nishida H, Kaida H, Ishibashi M, Baba K, Kouno K, Okuda S: Evaluation of exerciseinduced acute renal failure in renal hypouricemia using Tc-99m DTPA renography. Ann Nucl Med 2005;19:325-329.

25 Shima Y, Nozu K, Nozu Y, Togawa H, Kaito $\mathrm{H}$, Matsuo M, Iijima K, Nakanishi K, Yoshikawa N: Recurrent EIARF and PRES with severe renal hypouricemia by compound heterozygous SLC2A9 mutation. Pediatrics 2011; 127:e1621-e1625.

26 Fujinaga S, Ito A, Nakagawa M, Watanabe T, Ohtomo Y, Shimizu T: Posterior reversible encephalopathy syndrome with exercise-induced acute kidney injury in renal hypouricemia type 1. Eur J Pediatr 2013;172:15571560.

27 Price KL, Sautin YY, Long DA, Zhang L, Miyazaki $\mathrm{H}, \mathrm{Mu} \mathrm{W}$, Endou $\mathrm{H}$, Johnson RJ: $\mathrm{Hu}-$ man vascular smooth muscle cells express a urate transporter. J Am Soc Nephrol 2006;17: 1791-1795.

28 George J, Carr E, Davies J, Belch JJ, Struthers A: High-dose allopurinol improves endothelial function by profoundly reducing vascular oxidative stress and not by lowering uric acid. Circulation 2006;114:2508-2516.

29 Dinour D, Bahn A, Ganon L, Ron R, Geifman-Holtzman O, Knecht A, Gafter U, Rachamimov R, Sela BA, Burckhardt G, Holtzman EJ: URAT1 mutations cause renal hypouricemia type 1 in Iraqi Jews. Nephrol Dial Transplant 2011;26:2175-2181.

30 Wakasugi M, Kazama JJ, Narita I, Konta T, Fujimoto S, Iseki K, Moriyama T, Yamagata K, Tsuruya K, Asahi K, Kimura K, Kondo M, Kurahashi I, Ohashi Y, Watanabe T: Association between hypouricemia and reduced kidney function: a cross-sectional populationbased study in Japan. Am J Nephrol 2015;41: 138-146. 